PROCEEDINGS OF THE

AMERICAN MATHEMATICAL SOCIETY

Volume 127, Number 11, Pages 3345-3351

S 0002-9939(99)05217-X

Article electronically published on May 3, 1999

\title{
ON K-CONTACT MANIFOLDS WITH MINIMAL NUMBER OF CLOSED CHARACTERISTICS
}

\author{
PHILIPPE RUKIMBIRA
}

(Communicated by Christopher Croke)

\begin{abstract}
We prove that closed simply connected K-contact manifolds with minimal number of closed characteristics are homeomorphic to odd-dimensional spheres.
\end{abstract}

\section{INTRODUCTION}

A classical topological application of Morse theory states that an $n$-dimensional closed manifold admitting a Morse function with only 2 critical points is homeomorphic to the sphere $\mathbf{S}^{n}$. Since Morse theory has been extended into Morse-Bott theory with the notion of nondegenerate critical manifolds ([BOT]), it is quite natural to investigate the rigidity of the sphere using this generalized Morse theory. This is attempted in this paper, but only in the contact geometric setting.

Besides Smale's homological characterization of the sphere $\mathbf{S}^{n}, n \geq 5$, other "sphere theorems" have been obtained from some curvature restrictions (see [GOL], [XIA] and references in there). From the contact geometric point of view ([PAO]), the sphere has been characterized as the only hypersurface in $\mathbf{R}^{2 n}$ with codimension 1 isohelicoidal distributions. When nonintegrable, these distributions determine $\mathrm{K}$-contact forms. The novelty of Theorem 1 in this paper is that it requires no curvature hypothesis at all; it somehow complements the results in [GOL] where mainly regular contact manifolds are dealt with, but a K-contact manifold doesn't necessarily carry a regular contact structure.

Let's start with the basic definitions that will be needed throughout this paper. A contact form on a $2 n+1$-dimensional manifold $M$ is a 1 -form $\alpha$ such that the identity

$$
\alpha \wedge(d \alpha)^{n} \neq 0
$$

holds everywhere on $M$. Given such a 1-form, there is always a unique vector field $\xi$ satisfying $\alpha(\xi)=1$ and $i_{\xi} d \alpha=0$. The vector field $\xi$ is called the characteristic vector field of the contact form $\alpha$ and the corresponding 1-dimensional foliation is called a contact flow.

A contact form is called almost regular when its characteristic vector field is almost regular, that is: each point in $M$ belongs to a flow box pierced by the flow only a finite number of times. This implies that if $M$ is compact, all leaves

Received by the editors May 9, 1997 and, in revised form, January 12, 1998.

1991 Mathematics Subject Classification. Primary 58F05, 58F22; Secondary 53C15, 53C57.

Key words and phrases. K-contact form, clean function, closed characteristics. 
are circles and by a theorem of Wadsley ([WAD]), the leaves are orbits of a circle action on $M$. If this action is free, then the contact form is said to be regular.

Also, the contact manifold $(M, \alpha)$ admits a nonunique riemannian metric $g$ and a $(1,1)$ tensor field $J$ such that the following identities hold ([BLA]):

$$
\begin{gathered}
J \xi=0, \alpha(\xi)=1, J^{2}=-I+\alpha \otimes \xi, \alpha(X)=g(\xi, X), \\
g(J X, J Y)=g(X, Y)-\alpha(X) \alpha(Y), g(X, J Y)=d \alpha(X, Y) .
\end{gathered}
$$

The tensors $g, \alpha, \xi$ and $J$ will be referred to as structure tensors and $g$ is called a contact metric adapted to $\alpha$. When the characteristic vector field $\xi$ is Killing with respect to a contact metric $g$, then the manifold is said to be K-contact. Kcontact structures have been widely studied in [BLA] and one of their characteristic identities is

$$
\left(\nabla_{X} J\right) Y=R(\xi, X) Y
$$

where $R$ is the riemannian curvature tensor given by the expression:

$$
R(X, Y) Z=\nabla_{X} \nabla_{Y} Z-\nabla_{Y} \nabla_{X} Z-\nabla_{[X, Y]} Z .
$$

The odd dimensional sphere $\mathbf{S}^{2 n+1}$ carries K-contact forms with exactly $n+$ 1 closed characteristics; these are obtained from perturbations of the canonical sasakian form on $\mathbf{S}^{2 n+1}$ ([RU2]). We will prove that, in the simply connected category, the existence of a K-contact form whose contact flow has the minimum possible number of closed orbits characterizes the sphere up to homeomorphism.

\section{Morse theORY ON K-CONTACT MANifolds}

Let $\alpha, \xi, g$ be K-contact structure tensors on a closed manifold $M$ of dimension $2 n+1$. There exists a periodic Killing vector field $Z$ commuting with $\xi$ such that closed characteristics of $\alpha$ are exactly critical circles of the function $\mathbf{S}=\alpha(Z)$. In [RU1], Morse theory was carried out on sasakian closed manifold. Here, we present a similar application to K-contact, closed, not necessarily sasakian manifolds.

We now proceed to compute the hessian Hess $\mathbf{S}$ of $\mathbf{S}=\alpha(Z)$ in directions perpendicular to a connected component $N$ of the critical set of $\mathbf{S}$. Let $p \in N$ and $v, w$ be tangent vectors perpendicular to $N$ at $p$. We extend $v$ and $w$ into local vector fields $V$ and $W$ by parallel translation along geodesics emanating from $p$. In particular, the identities $(\nabla V)(p)=0=(\nabla W)(p)$ are valid and will be used repeatedly as well as the identity $\nabla_{X} \xi=-J X$ valid on any $\mathrm{K}$-contact manifold ([BLA]). The fact that $Z$ is a Killing vector field commuting with $\xi$ will also be used.

$$
\begin{aligned}
\operatorname{Hess}_{\mathbf{S}}(p)(v, w) & =(V(W g(\xi, Z)))(p) \\
& =\left(V\left(-g(J W, Z)+g\left(\xi, \nabla_{W} Z\right)\right)\right)(p) \\
& =\left(-g\left(\nabla_{V} J W, Z\right)-g\left(J W, \nabla_{V} Z\right)-V g\left(\nabla_{\xi} Z, W\right)\right)(p) \\
& =\left(-g\left(\nabla_{V} J W, Z\right)-g\left(J W, \nabla_{V} Z\right)+V g(J Z, W)\right)(p) \\
& =\left(-g\left(\nabla_{V} J W, Z\right)-g\left(J W, \nabla_{V} Z\right)+g\left(\nabla_{V} J Z, W\right)\right.
\end{aligned}
$$




$$
\begin{aligned}
& \left.+g\left(J Z, \nabla_{V} W\right)\right)(p) \\
& =\left(-g\left(\left(\nabla_{V} J\right) W, Z\right)-g\left(J \nabla_{V} W, Z\right)-g\left(J W, \nabla_{V} Z\right)\right. \\
& \left.+g\left(\left(\nabla_{V} J\right) Z, W\right)+g\left(J \nabla_{V} Z, W\right)+g\left(J Z, \nabla_{V} W\right)\right)(p) \\
& =\left(-g(R(\xi, V) W, Z)+2 g\left(\nabla_{V} W, J Z\right)+2 g\left(W, J \nabla_{V} Z\right)\right. \\
& +g(R(\xi, V) Z, W))(p) \text { by identity }(1) \\
& =\left(2 g(R(\xi, V) Z, W)+2 g\left(W, J \nabla_{V} Z\right)\right)(p) .
\end{aligned}
$$

Evaluating at $p$, we have obtained the identity:

$$
\operatorname{Hess}_{\mathbf{S}}(p)(v, w)=2 g(R(\xi, v) Z, w)+2 g\left(w, J \nabla_{v} Z\right) .
$$

Let $\alpha(Z)(p)=k$ and put $Z=k \xi+\delta$ where $\delta$ is a Killing vector field vanishing all along $N$. Then points at which $\delta$ is zero lie on periodic orbits of $\xi$ and one has the identity:

$$
\nabla_{v} Z=-k J v+\nabla_{v} \delta
$$

Lemma 1. (1) The tangent vector $\nabla_{v} \delta$ is nonzero and perpendicular to $N$.

(2) The hessian Hess $\mathbf{S}$ of $\alpha(Z)$ along $N$ satisfies

$$
\operatorname{Hess}_{\mathbf{S}}(p)\left(v, J \nabla_{v} \delta\right)=2 g\left(\nabla_{v} \delta, \nabla_{v} \delta\right)
$$

and is therefore nondegenerate in directions perpendicular to $N$.

Proof. The proof of assertion (1) is the same as in [RU1].

Now combining identities (2) and (3) and exploiting the tensor nature of $R$ as well as the identity $R(\xi, v) \xi=-v$ ([BLA], page 65$)$ we see that:

$$
\begin{aligned}
\operatorname{Hess}_{\mathbf{S}}(p)\left(v, J \nabla_{v} \delta\right) & =2 g\left(R(\xi, v) Z, J \nabla_{v} \delta\right)+2 g\left(J \nabla_{v} Z, J \nabla_{v} \delta\right) \\
& =2 k g\left(R(\xi, v) \xi, J \nabla_{v} \delta\right)+2 g\left(J \nabla_{v} Z, J \nabla_{v} \delta\right) \\
& =-2 k g\left(v, J \nabla_{v} \delta\right)+2 g\left(J \nabla_{v} Z, J \nabla_{v} \delta\right) \\
& =2 k g\left(J v, \nabla_{v} \delta\right)+2 g\left(\nabla_{v} \delta-k J v, \nabla_{v} \delta\right) \\
& =2 k g\left(J v, \nabla_{v} \delta\right)+2 g\left(\nabla_{v} \delta, \nabla_{v} \delta\right) \\
& -2 k g\left(J v, \nabla_{v} \delta\right)=2 g\left(\nabla_{v} \delta, \nabla_{v} \delta\right) \neq 0 .
\end{aligned}
$$

It follows from $\operatorname{Hess}_{\mathbf{S}}(p)\left(v, J \nabla_{v} \delta\right) \neq 0$ that $\operatorname{Hess}_{\mathbf{S}}(p)$ is nondegenerate in directions perpendicular to $N$, which completes the proof of Lemma 1.

In general, for a smooth function $f$, the normal bundle $\nu N$ of a nondegenerate critical manifold $N$ is decomposed into positive and negative parts:

$$
\nu N=\nu^{+} N \oplus \nu^{-} N
$$

where $\nu_{p}^{+} N$ and $\nu_{p}^{-} N$ are the positive and negative eigenspaces of the hessian of $f$, Hess $_{f}$. The fiber dimension of $\nu^{-} N$, denoted by $\lambda_{N}$, is referred to as the index of $N$ relative to $f$. A function $f$ all of whose critical manifolds are nondegenerate is said to be a clean function. For a proof of the following proposition, we refer to [GST].

Proposition 1. Let $f: X \rightarrow \mathbf{R}$ be a clean function. Suppose that the indices of all critical manifolds of $f$ are even. Then $f$ has a unique local maximum. 
On a compact manifold $X$, Proposition 1 implies that a clean function has a unique minimum and a unique maximum.

Let $\theta^{-}$denote the orientation bundle of $\nu^{-} N$ and

$$
P_{t}(M ; \mathbf{R})=\sum t^{k} \operatorname{dim} H^{k}(M ; \mathbf{R})
$$

the Poincaré series of $M$. If one defines the Morse series ([BOT]) of a clean function $f$ relative to the coefficient field $\mathbf{R}$ by:

$$
\mathcal{M}_{t}(f)=\sum t^{\lambda_{N}} P_{t}\left(N ; \theta^{-} \otimes \mathbf{R}\right)
$$

where the sum runs over all critical manifolds of $f$, then the Morse inequalities hold:

$$
\mathcal{M}_{t}(f) \geq P_{t}(M ; \mathbf{R}) .
$$

The inequality (5) implies that $\mathcal{M}_{t}(f)$ majorizes $P_{t}(M ; \mathbf{R})$ coefficient by coefficient.

Now as in [RU1], we have the following:

Proposition 2. The function $\mathbf{S}=\alpha(Z)$ is clean and each of its critical manifolds has even index.

Proof. By Lemma 1, each critical submanifold of $\alpha(Z)$ is nondegenerate. It remains to prove the assertion about even index. To that end, let $v$ be any direction perpendicular to $N$ at $p$. Then, using identity (2), we obtain:

$$
\begin{aligned}
\operatorname{Hess}_{\mathbf{S}}(p)(J v, J v) & =2 g(R(\xi, J v) Z, J v)+g\left(J \nabla_{J v} Z, J v\right) \\
& =-2 k g(J v, J v)-2 g\left(J v, \nabla_{v} Z\right) \\
& =-2 k g(v, v)+2 g\left(v, J \nabla_{v} Z\right) \\
& =2 g(R(\xi, v) Z, v)+2 g\left(v, J \nabla_{v} Z\right)=\operatorname{Hess}_{\mathbf{S}}(p)(v, v) .
\end{aligned}
$$

This clearly establishes the fact that if $\mathrm{Hess}_{\mathbf{S}}$ is negative definite in the direction $v$, it is also negative definite in the direction $J v$; hence the indices are all even.

Assume now that the closed K-contact $2 n+1$-dimensional manifold $M$ with a function $\alpha(Z)$ as in Proposition 1 has a finite number $n+1$ of closed characteristics, one for each even index $0,2, \ldots, 2 n$. Let's denote the critical circles by $N_{i}$,

$$
\mathcal{M}_{t}(\alpha(Z))=\sum_{i=0}^{n} t^{\lambda_{N_{i}}} P_{t}\left(N_{i} ; \theta_{i}^{-} \otimes \mathbf{R}\right)
$$

where $\lambda_{N_{i}}=2 i$ are even integers. But since for $i=0,1,2, \ldots, n, \nu^{-} N_{i}$ is an orientable vector bundle over $\mathbf{S}^{1}$, one has:

$$
P_{t}\left(N_{i} ; \theta_{i}^{-} \otimes \mathbf{R}\right)=1+t .
$$

Hence, using the notation $b_{i}(M)$ for the dimension of $H^{i}(M ; \mathbf{R})$, the Morse inequalities (5) can be written as:

$$
\sum_{i=0}^{n} t^{\lambda_{N_{i}}}(1+t) \geq \sum_{i=0}^{2 n+1} b_{i}(M) t^{i}
$$

As a result, one has the following theorem:

Theorem 1. Let $M^{2 n+1}$ be a closed $K$-contact manifold with $n+1$ closed characteristics. Then each of the real Betti numbers of $M^{2 n+1}$ is less or equal to 1. 


\section{Stratification FOR K-CONTACT MANifOldS}

Given a K-contact manifold $M^{2 n+1}$ with $n+1$ closed characteristics, $N_{0}, N_{1}, \ldots$, $N_{n}$ which are critical circles of a function $\alpha(Z)$ with index $0,2, \ldots, 2 n$ respectively, we shall denote by $M^{2 k+1}, 0 \leq k \leq n$, the closures of the sets $\left\{x \in M, \lim _{t \rightarrow \infty} \Psi_{t}(x) \in\right.$ $\left.N_{k}\right\}$, where $\Psi_{t}$ is the gradient flow for the function $\alpha(Z)$. For an arbitrary vector field $Y$ on $M^{2 n+1}$, one has

$$
d(\alpha(Z))(Y)=d i_{Z} \alpha(Y)=g(J Z, Y) .
$$

Therefore, $\Psi_{t}$ is generated by the vector field $J Z$. For the argument to follow, we point out that $\xi, Z$ and $J Z$ are commuting vector fields. The subsets $M^{2 k+1}$ constitute a stratification of $M^{2 n+1}$ as follows:

$$
M^{1} \subset M^{3} \subset M^{5} \subset \ldots \subset M^{2 n-1} \subset M^{2 n+1}
$$

and each $M^{2 k+1}$ is a closed $2 k+1$-dimensional submanifold with $k+1$ nondegenerate critical circles for the restriction of $\alpha(Z)$.

Proposition 3. $M^{3}$ is a minimal submanifold, which is diffeomorphic to a quotient of the standard $\mathbf{S}^{3}$ by an abelian finite group of isometries.

Proof. For $x \in N_{0}$ or $x \in N_{1}$, if $v \in T_{x} M^{3}$ and $\alpha(v)=0$, then the fact that $J v \in T_{x} M^{3}$ follows from $J$-invariance of the splitting $\nu_{N_{i}}=\nu_{N_{i}}^{-} \oplus \nu_{N_{i}}^{+}$for the normal bundle of $N_{i}, i=0,1$ (see Proposition 2). For $x \in M^{3}-\left(N_{0} \cup N_{1}\right)$, the tangent space $T_{x} M^{3}$ is spanned by the vectors $J Z, \xi$ and $Z-\alpha(Z) \xi$ and is therefore $J$-invariant. This implies that $M^{3}$ is a K-contact invariant submanifold and it is known that contact invariant submanifolds are minimal ([END]).

Since $M^{3}$ supports an isometric flow with exactly two closed orbits, it follows from Carrière's classification of riemannian flows on compact 3-manifolds ([CAR]), that $M^{3}$ is diffeomorphic to either a quotient of $\mathbf{S}^{3}$ under an abelian finite group of isometries or $\mathbf{S}^{2} \times \mathbf{S}^{1}$. But $H^{1}\left(M^{3}\right)=0$ ([RU1]) and hence $M^{3}$ must be diffeomorphic to a lens space.

Remark. It is possible that each $M^{2 k+1}$ is a contact invariant, hence minimal submanifold of $M^{2 n+1}$.

\section{A Characterization of the SPhere}

The subsets $M^{2 k-1}$ and $N_{k}$ are disjoint closed submanifolds of $M^{2 k+1}$, so each has a tubular neighborhood in $M^{2 k+1}$. In fact, one can find open neighborhoods $U$ of $M^{2 k-1}$ and $V$ of $N_{k}$ such that $M^{2 k-1}, N_{k} \simeq \mathbf{S}^{1}$ and $\mathbf{S}^{2 k-1} \times \mathbf{S}^{1}$ are deformation retracts of $U, V$ and $U \cap V$ respectively. The Mayer-Vietoris sequence for the homology yields the following long exact sequence:

$$
\begin{aligned}
\ldots \rightarrow H_{*}\left(\mathbf{S}^{2 k-1} \times \mathbf{S}^{1}\right) \rightarrow H_{*}\left(M^{2 k-1}\right) \oplus H_{*}\left(\mathbf{S}^{1}\right) & \rightarrow H_{*}\left(M^{2 k+1}\right) \\
& \rightarrow H_{*-1}\left(\mathbf{S}^{2 k-1} \times \mathbf{S}^{1}\right) \rightarrow \ldots
\end{aligned}
$$

Lemma 2. If $H_{1}\left(M^{2 k+1}\right)=0, k \geq 2$, then $H_{1}\left(M^{2 k-1}\right)=0$ and $H_{2}\left(M^{2 k-1}\right) \simeq$ $\mathrm{H}_{2}\left(M^{2 k+1}\right)$.

Proof. Writing the exact sequence (6) from $*=2$ down to $*=1$ yields:

$$
0 \rightarrow H_{2}\left(M^{2 k-1}\right) \oplus 0 \rightarrow H_{2}\left(M^{2 k+1}\right) \rightarrow \mathbf{Z} \rightarrow H_{1}\left(M^{2 k-1}\right) \oplus \mathbf{Z} \rightarrow 0 \rightarrow \ldots
$$

Lemma 2 follows from exactness of the above sequence. 
Lemma 3. If $H_{1}\left(M^{2 k+1}\right)=0$ and $M^{2 k-1}$ is a homology sphere, then $M^{2 k+1}$ is also a homology sphere.

Proof. We need only to prove $H_{i}\left(M^{2 k+1}\right)=0$ for $2<i \leq k$, the case $i=2$ being covered by Lemma 2 and the cases $i>k$ will follow from duality.

Writing the sequence (6) for $2<i \leq k$ yields the following:

$$
\ldots \rightarrow 0 \rightarrow 0 \rightarrow 0 \oplus 0 \rightarrow H_{i}\left(M^{2 k+1}\right) \rightarrow 0 \rightarrow \ldots .
$$

Therefore $H_{i}\left(M^{2 k+1}\right)=0$ for $2<i \leq k$. Now by duality, $H_{2 k+1-i}\left(M^{2 k+1}\right)=0$ for $2 \leq i \leq k$. This completes the proof of Lemma 3 .

Theorem 2. Every closed simply connected $K$-contact manifold $M^{2 n+1}$ with a finite number $n+1$ of closed characteristics is homeomorphic to the sphere $\mathbf{S}^{2 n+1}$.

Proof. If $\pi_{1}\left(M^{2 n+1}\right)=0$, then $H_{1}\left(M^{2 n+1}\right)=0$ and by Lemma $2, H_{1}\left(M^{2 k+1}\right)=0$ for $1 \leq k \leq n$. Since $M^{3}$ is a homology sphere, it follows from Lemma 3 and induction on $k$ that $M^{2 n+1}$ is a homology sphere and thus, by Smale's solution to the generalized Poincaré conjecture ([SMA]), $M^{2 n+1}$ is homeomorphic to $\mathbf{S}^{2 n+1}$ for $n \geq 2$. The case $n=1$ is covered by Proposition 3 .

Remark. We have actually proved that if $M^{2 n+1}$ is simply connected, then each of $M^{2 k+1}, 1 \leq k \leq n$, is homeomorphic to $\mathbf{S}^{2 k+1}$.

\section{Application to BRieskorn manifolds}

Let $N \in \mathbf{C}^{3}$ be a complex submanifold of complex dimension 2 and $\mathbf{S}^{5}$ the standard sphere in complex 3 -space. Suppose $N$ intersects $\mathbf{S}^{5}$ transversally in a differentiable manifold $M=N \cap \mathbf{S}^{5}$. Then the restriction of the standard contact form on $\mathbf{S}^{5}$ to $M$ is a contact form on $M$ ([ABE]). Assume now that the origin $\mathbf{o}$ is an isolated singularity of a polynomial $f\left(z_{1}, z_{2}, z_{3}\right)$ and consider the compact 3dimensional manifold $N=f^{-1}(0) \cap \mathbf{S}_{\epsilon}^{5}$, where $\mathbf{S}_{\epsilon}^{5}$ is the sphere of radius $\epsilon$, centered at o. In $[\mathrm{ABE}]$, it has been announced that these contact manifolds $M$ are often nonregular and examples of polynomials $f$ for which $\pi_{1}(M)$ is finite nonabelian have been exhibited in there. Nevertheless, we prove that they are almost regular.

Theorem 3. Let $M$ be a manifold which is a transverse intersection of $f^{-1}(0)$ with $\mathbf{S}_{\epsilon}^{5}$, where $f$ is a (generalized) Brieskorn polynomial with isolated singularity at the origin o of $\mathbf{C}^{3}$ and $\mathbf{S}_{\epsilon}^{5}$ is the sphere of small radius $\epsilon$, centered at $\mathbf{o}$. If $\pi_{1}(M)$ is not abelian, then any $K$-contact form on $M$ is almost regular.

Proof. Suppose $M$ has a K-contact form which is not almost regular. By the structure Theorem 2.1 in [RU3] and Proposition 2 of this paper, $M$ would carry a finite number of closed characteristics. By Proposition 1, there are exactly 2 closed characteristics which are critical circles of a clean function, one of index 0 and another one of index 2. Hence by Proposition 3, $M$ would be homeomorphic to a quotient of $\mathbf{S}^{3}$ under an abelian finite group of isometries, contradicting the hypothesis that $\pi_{1}\left(M^{3}\right)$ is not abelian.

\section{ACKNOWLEDGMENT}

The author would like to thank the referee for his/her suggestions about improving the presentation of this paper. 


\section{REFERENCES}

[ABE] Abe, K. and Erbacher, J., Nonregular contact structures on Brieskorn manifolds, Bull. Amer. Math. Soc. 81 (1975), 407-409. MR 54:6019

[BLA] Blair, D., Contact manifolds in riemannian geometry, Springer Lectures Notes in Math, vol. 509, Springer Verlag, Berlin and New York, 1976. MR 57:7444

[BOT] Bott, R., Lectures on Morse Theory, old and new, Bulletin (New series) of the AMS 7 (1982), 331-358. MR 84m:58026a

[CAR] Carrière, Y., Flots riemanniens, Structures transverses des feuilletages, Astérisque 116 (1982), 31-52. MR 86m:58125a

[END] Endo, H., Invariant submanifolds in a contact riemannian manifold, Tensor (N.S.) 42 (1985), 86-89. MR 87e:53087

[GOL] Goldberg, S.I., Nonnegatively curved contact manifolds, Proc. Amer. Math. Soc. 96 (1986), 651-656. MR 87d:53068

[GST] Guillemin, V. and Sternberg, S., Convexity Properties of the Moment Mapping, Invent. math. 67 (1982), 491-513. MR 83m:58037

[PAO] Piu, P., Sur une charactérisation de la sphère $\mathbf{S}^{2 n+1}$ en termes de géométrie riemannienne de contact, Rendic. Cir. Math. Pal. Tomo XXXVIII (1989), 297-304.

[RU1] Rukimbira, P., Topology and closed characteristics on K-contact manifolds, Bull. Belg. Math. Soc. 2 (1995), 349-356. MR 96g:53044

[RU2] Rukimbira, P., Chern-Hamilton conjecture and K-contactness, Houston J. Math. 21 (1995), 709-718. MR 96m:53032

[RU3] Rukimbira, P., Some remarks on R-contact flows, Ann. Global Analy. Geom. 11, no 3 (1993), 165-171. MR 94h:53043

[SMA] Smale, S., The generalized Poincaré conjecture in higher dimensions, Bull. Amer. Math. Soc. (N.S.) 66 (1960), 373-375. MR 23:A2220

[WAD] Wadsley, A.W., Geodesic foliations by circles, J. Diff. Geom. 10 (1975), 541-549. MR 53:4092

[XIA] Xia, C., A generalization of the classical sphere theorem, Proc. Amer. Math. Soc. 125 (1997), 255-258. MR 98h:53059

Department of Mathematics, Florida International University, Miami, Florida 33199

E-mail address: rukim@fiu.edu 